\title{
Optimal Weight Vectors for Broadcast Channels*
}

\author{
Murat Torlak, Guanghan Xu, and Brian L. Evans \\ Dept. of Electrical \& Computer Engineering \\ The Univ. of Texas at Austin \\ Austin, TX 78712-1084
}

\author{
Hui Liu \\ Department of Electrical Engineering \\ University of Virginia \\ Charlottesville, VA 22903-2442
}

\begin{abstract}
In a multi-transmitter broadcast system, the weight vector for each message signal can provide an additional degree-of-freedom for signal enhancement and interference suppression by taking advantage of the spatial diversity among the users. To date, the design of the optimal weight vectors which maximize the overall channel capacity is still an open problem. The main reason is attributed to the fact that under certain power constraints, the channel capacity $R$ is a highly nonlinear function of the $M$-dimensional weight vectors $\left\{\mathbf{w}_{i}\right\}$, where $M$ is the number of transmitters. Therefore, the maximization of $R$ over $\left\{\mathbf{w}_{i}\right\}$ does not seem to be tractable to simple mathematic treatment. In this paper, we present our studies on designing optimal weight vectors for a two-user, multi-transmitter broadcast channel. Our primary contribution is to decouple the weight vectors in $R$ which simplifies the optimization problem to a search for the maxima of a smooth two-dimensional function.
\end{abstract}

\section{Introduction}

The single-transmitter broadcast channels were studied by Cover in [4]. Since Cover's novel work, increased demand for wireless communications and mobile cellular communications has motivated to incorporate the use of spatial diversity to the existing systems. Both multi-transmitter and multi-receiver systems are being explored to increase the capacity and improve the performance of wireless communications $[3,1]$. In this paper, we focus on the multitransmitter broadcast system. The multi-transmitter

\footnotetext{
*This work was sponsored in part by NSF CAREER A ward under Grant MIP-9502695, Office of Naval Research under Grant N00014-95-1-0638, the Joint Services Electronics Program under Contract F49620-95-C-0045, Motorola, Inc., Southwestern Bell Technology Resources, Inc. and Texas Instruments. The United States Government is authorized to reproduce and distribute reprints for governmental purposes notwithstanding any copyright notation hereon.
}

broadcast channel is a communication channel in which there is a transmitting antenna array system and two or more receiving users. The basic problem is to find the optimum weight vector for each message signal to maximize the overall channel capacity. In particular, We study the channel capacity assuming only independent deconding since it is more feasable to implement for real applications than the joint decoding.

Some recent investigations on wireless communication systems with the use of an antenna array, i.e, Spatial-Diversity-Multiple-Access (or SDM A) systems $[2,5,6]$, have attempted to exploit spatial diversity among the users. However, the design of the optimal weight vectors which maximize the overall channel capacity of the broadcast channels is still an open problem. The primary reason is attributed to the fact that under certain power constraints, the channel capacity $R$ is a highly nonlinear function of the $M$-dimensional weight vectors $\left\{\mathbf{w}_{i}\right\}$, where $M$ is the number of transmitters. Therefore, the goal of this paper is to present our studies on the optimal weight vector design problem for a two-user, multi-transmitter broadcast channel. Our studies on the channel capacity based on only a simple two-user system may seem restrictive; however, our motivation was the fact that this simple model can provide insights on multi-user broadcast channals.

\section{Background}

We consider maximizing the channel capacity in a two-user broadcast system with multiple transmitters. Letting $s_{1}$ and $s_{2}$ be the message signals for the first and second users, the base station weights each signal with a weight vector and then transmits the superimposed signal from an array with $M$ elements:

$$
\mathbf{y}(t)=\beta_{1} \mathbf{w}_{1} s_{1}(t)+\beta_{2} \mathbf{w}_{2} s_{2}(t) .
$$

The signals $s_{1}(t)$ and $s_{2}(t)$ are assumed to be i.i.d. with Gaussian distribution; $\mathbf{w}_{1}$ and $\mathbf{w}_{2}$ are normalized weight vectors, i.e., $\left\|\mathbf{w}_{i}\right\|=1, i=1,2 ; \beta_{1}$ and $\beta_{2}$ are 
the transmitting magnitudes which are subjected to certain power constraints. For simplicity, we assume

$$
\beta_{1}^{2}+\beta_{2}^{2}=1 \text {. }
$$

If independent decoding is employed at the user receivers, the achievable channel capacities can be expressed as

$$
\begin{aligned}
& R_{1}=\frac{1}{2} \log \left(1+\frac{\alpha_{1}^{2} \beta_{1}^{2} \mathbf{w}_{1}^{H} \mathbf{a}_{1} \mathbf{a}_{1}^{H} \mathbf{w}_{1}}{1+\alpha_{1}^{2} \beta_{2}^{2} \mathbf{w}_{2}^{H} \mathbf{a}_{1} \mathbf{a}_{1}^{H} \mathbf{w}_{2}}\right) \\
& R_{2}=\frac{1}{2} \log \left(1+\frac{\alpha_{2}^{2} \beta_{2}^{2} \mathbf{w}_{2}^{H} \mathbf{a}_{2} \mathbf{a}_{2}^{H} \mathbf{w}_{2}}{1+\alpha_{2}^{2} \beta_{1}^{2} \mathbf{w}_{1}^{H} \mathbf{a}_{2} \mathbf{a}_{2}^{H} \mathbf{w}_{1}}\right) .
\end{aligned}
$$

where $\mathbf{a}_{i}=\left(\begin{array}{llll}a_{i, 1} & a_{i, 2} & \cdots & a_{i, M}\end{array}\right)^{T}$ such that $\left\|\mathbf{a}_{i}\right\|=1$ is the spatial signature which represents the propagation pattern of the $i^{t h}$ user. $\alpha_{i}$ is its associated magnitude. The noise power has been normalized to unity.

Due to the additional degrees of freedom introduced by multiple transmitters, one can manipulate the complex weight vectors to enhance the desired signal and at the same time, suppress the interference. In fact, there exists $\mathbf{w}_{i}, \mathbf{w}_{i}^{H} \mathbf{a}_{i}=\delta_{i j}$ which can completely eliminate the interference from one to the other. However, such a selection may not be optimal in terms of overall performance, e.g., as measured by $R=R_{1}+R_{2}$, since the desired signal power at the receiver may be reduced as well.

\subsection{Single Antenna}

If we select

$$
\mathbf{w}_{1}=\mathbf{w}_{2}=\left(\begin{array}{llllll}
0 & \cdots & 0 & 1 & 0 & \cdots
\end{array}\right)^{T}
$$

the system reduces to using a single antenna, and the signals received by the first and second users become $\alpha_{1} \mathbf{a}_{1}(k)\left(\beta_{1} s_{1}(t)+\beta_{2} s_{2}(t)\right)$ and $\alpha_{2} \mathbf{a}_{2}(k)\left(\beta_{1} s_{1}(t)+\right.$ $\left.\beta_{2} s_{2}(t)\right)$, respectively. The capacities are given by

$$
\begin{aligned}
& R_{1}=\frac{1}{2} \log \left(1+\frac{\beta_{1}^{2} \alpha_{1}^{2}\left|\mathbf{a}_{1}(k)\right|^{2}}{1+\beta_{2}^{2} \alpha_{1}^{2}\left|\mathbf{a}_{1}(k)\right|^{2}}\right) ; \\
& R_{2}=\frac{1}{2} \log \left(1+\frac{\beta_{2}^{2} \alpha_{2}^{2}\left|\mathbf{a}_{2}(k)\right|^{2}}{1+\beta_{1}^{2} \alpha_{2}^{2}\left|\mathbf{a}_{2}(k)\right|^{2}}\right) .
\end{aligned}
$$

Since in general, $\mathbf{a}_{i}(k)<1$, the omni-directional transmission pattern wastes much energy in free space, and the above formulation is obviously not optimal.

\subsection{Naive Time Sharing Retransmission}

Consider the case when $\beta_{1}=1$ and $\beta_{2}=0$. The optimal weight vector that focuses all the energy to the first user is clearly $\mathbf{w}_{1}=\mathbf{a}_{1}$, which gives $R_{1}=$ $\frac{1}{2} \log \left(1+\alpha_{1}^{2}\right)$. On the other hand, if $\beta_{1}=0$ and $\beta_{2}=1, \mathbf{w}_{2}$ should be selected as $\mathbf{a}_{2}$, which gives $R_{2}=$ $\frac{1}{2} \log \left(1+\alpha_{2}^{2}\right)$. Using the naive time sharing scheme, the resulting capacity is plotted in Figure 2.

\subsection{Orthogonal Spatial Channels}

In this case, $\mathbf{w}_{i}^{H} \mathbf{a}_{j}=\delta(i, j)$ which forms an orthogonal channel in which each message can be transmitted without interference. As shown in Figure $1, \mathbf{w}_{1}$ should be in the direction of $\mathbf{P} \mathbf{a}_{2} \mathbf{a}_{1}$, where $\mathbf{P} \frac{\perp}{\mathbf{a}_{2}}$ denotes the projection operator to the orthogonal space of $\mathbf{a}_{2}$.

We use $R=R_{1}+R_{2}$ as a performance measure. The design of optimal weight vectors is a nontrivial problem due to the involvement of high-dimensional quantities, and more importantly, the nonlinearity of $R$ in terms of $\mathbf{w}_{1}$ and $\mathbf{w}_{2}$.

\section{Dimension Reduction and Decou- pling}

For a given pair of $\left(\beta_{1}, \beta_{2}\right)$, our goal is to find the optimal weight vectors $\mathbf{w}_{1}$ and $\mathbf{w}_{2}$ such that the total capacity $R=R_{1}+R_{2}$ is maximized. Denote

$$
\begin{aligned}
& \kappa_{11}=\mathbf{w}_{1}^{H} \mathbf{a}_{1} \mathbf{a}_{1}^{H} \mathbf{w}_{1} ; \\
& \kappa_{21}=\mathbf{w}_{1}^{H} \mathbf{a}_{2} \mathbf{a}_{2}^{H} \mathbf{w}_{1} ; \\
& \kappa_{22}=\mathbf{w}_{2}^{H} \mathbf{a}_{2} \mathbf{a}_{2}^{H} \mathbf{w}_{2} . \\
& \kappa_{12}=\mathbf{w}_{2}^{H} \mathbf{a}_{1} \mathbf{a}_{1}^{H} \mathbf{w}_{2} .
\end{aligned}
$$

where $\kappa_{i j}=\cos ^{2} \angle\left(\mathbf{w}_{i}, \mathbf{a}_{j}\right)$ is a measure of the angle between the $i$ th weight vector and the $j$ th spatial signature vector. Thus it is confined to $[0,1]$. It is seen from (1) that for certain $\kappa_{11}$ and $\kappa_{22}, R$ is optimal if $\kappa_{21}=\mathbf{w}_{1}^{H} \mathbf{a}_{2} \mathbf{a}_{2}^{H} \mathbf{w}_{1}=\cos ^{2} \angle\left(\mathbf{w}_{1}, \mathbf{a}_{2}\right)$ and $\kappa_{12}=\mathbf{w}_{2}^{H} \mathbf{a}_{1} \mathbf{a}_{1}^{H} \mathbf{w}_{2}=\cos ^{2} \angle\left(\mathbf{w}_{2}, \mathbf{a}_{1}\right)$ are minimized. The focus of the problem is to find the maximum values of $\kappa_{11}$ and $\kappa_{22}$ in terms of $\kappa_{21}$ and $\kappa_{12}$ or to find the minimum values of $\kappa_{21}$ and $\kappa_{12}$ in terms of $\kappa_{11}$ and $\kappa_{22}$, respectively. This can be formulated as follows

$$
\begin{aligned}
& \min _{\left\|\mathbf{w}_{1}^{H} \mathbf{a}_{1}\right\|^{2}=\kappa_{11},\left\|\mathbf{w}_{1}\right\|=1}\left\|\mathbf{w}_{1}^{H} \mathbf{a}_{2}\right\|^{2} ; \\
& \min _{\left\|\mathbf{w}_{2}^{H} \mathbf{a}_{2}\right\|^{2}=\kappa_{22},\left\|\mathbf{w}_{2}\right\|=1}\left\|\mathbf{w}_{2}^{H} \mathbf{a}_{1}\right\|^{2} .
\end{aligned}
$$

Note that in the above optimization problem, $\mathbf{w}_{1}$ and $\mathbf{w}_{2}$ are no longer interwined and thus can be solved separately. In the remainder of this paper, we shall only address the problem defined in (4), and the optimization of $\mathbf{w}_{2}$ can be carried in the same fashion.

\section{Optimization}

To solve this problem in (4), we use the method of Lagrange multipliers. Defining $\Phi\left(\lambda_{1}, \lambda_{2}, \mathbf{w}_{1}\right)$ as

$\mathbf{w}_{1}^{H} \mathbf{a}_{2} \mathbf{a}_{2}^{H} \mathbf{w}_{1}+\lambda_{1}\left(\kappa_{11}-\mathbf{w}_{1}^{H} \mathbf{a}_{1} \mathbf{a}_{1}^{H} \mathbf{w}_{1}\right)+\lambda_{2}\left(1-\mathbf{w}_{1}^{H} \mathbf{w}_{1}\right)$ 
where $\lambda_{1}$ and $\lambda_{2}$ are real numbers. We see that the equation $\partial \Phi / \partial \mathbf{w}_{1}=\mathbf{0}$ leads to the linear system

$$
\begin{aligned}
\partial \Phi / \partial \mathbf{w}_{1} & =\mathbf{a}_{2} \mathbf{a}_{2}^{H} \mathbf{w}_{1}-\lambda_{1} \mathbf{a}_{1} \mathbf{a}_{1}^{H} \mathbf{w}_{1}-\lambda_{2} \mathbf{w}_{1} \\
& =\underbrace{\left(\mathbf{a}_{2} \mathbf{a}_{2}^{H}-\lambda_{1} \mathbf{a}_{1} \mathbf{a}_{1}^{H}-\lambda_{2} \mathbf{I}\right)}_{\mathbf{A}} \mathbf{w}_{1}=0 .
\end{aligned}
$$

Thus, $\mathbf{w}_{1}$ must be one of the eigenvectors of $\mathbf{A}$ which corresponds to a zero eigenvalue of $\mathbf{A}$.

\section{$4.1 \quad \lambda_{2}=0$}

When $\lambda_{2}=0, \lambda_{1}$ must be equal to zero, since otherwise $\mathbf{a}_{1}^{H} \mathbf{w}_{1}=0$, which conflicts with $\mathbf{w}_{1}^{H} \mathbf{a}_{1} \mathbf{a}_{1}^{H} \mathbf{w}_{1}=$ $\kappa_{11}$. Therefore, the problem becomes equivalent to finding a $\mathbf{w}_{1}$ such that $\mathbf{w}_{1}^{H} \mathbf{a}_{2}=0$ subject to $\mathbf{w}_{1}^{H} \mathbf{a}_{1} \mathbf{a}_{1}^{H} \mathbf{w}_{1}=\kappa_{11}$. The following Theorem gives the answer.

Theorem 1 Let $\mathbf{P} \grave{\mathbf{a}}_{2}$ to the projection of the null space of $\mathbf{a}_{2}$. There exists at least one $\mathbf{w}_{1}$ such that $\mathbf{w}_{1}^{H} \mathbf{a}_{2}=0$ subject to $\mathbf{w}_{1}^{H} \mathbf{a}_{1} \mathbf{a}_{1}^{H} \mathbf{w}_{1}=\kappa_{11}$, provided that $\mathbf{w}_{1}^{H} \mathbf{P} \frac{\perp}{\mathbf{a}_{2}} \mathbf{w}_{1} \geq \kappa_{11}$.

The above theorem is intuitive from the graphic illustration in Figure 1. Those weight vectors which satisfy $\mathbf{w}_{1}^{H} \mathbf{a}_{1} \mathbf{a}_{1}^{H} \mathbf{w}_{1}=\kappa_{11}$ can be visualized as a cone in a high-dimensional space, since the $\mathbf{w}_{1}^{H} \mathbf{a}_{1} \mathbf{a}_{1}^{H} \mathbf{w}_{1}$ term is basically the norm-squared of the projection of $\mathbf{w}_{1}$ onto $\mathbf{a}_{1}$. To assure $\mathbf{w}_{1}^{H} \mathbf{a}_{2}=0, \mathbf{w}_{1}$ must be in the null space of $\mathbf{a}_{2}$. This can only happen if the cone intersects the null space; in other words, the projection of $\mathbf{a}_{1}$ onto $\mathbf{P}_{\mathbf{a}_{2}}^{\perp}$ is great than $\kappa_{1}$. A simple proof follows, Proof:

Let $\mathbf{a}_{1,2}^{\perp}$ be a vector which is orthogonal to both $\mathbf{a}_{1}$ and $\mathbf{a}_{2}$ and $\mathbf{P} \mathbf{a}_{2} \mathbf{a}_{1}$ be the projection of $\mathbf{a}_{1}$ in $\mathbf{P} \mathbf{a}_{2}$. Let $\mathbf{v}$ be a vector pointing from the origin toward a point on the line connecting $\mathbf{a}_{1,2}^{\perp}$ and $\mathbf{P}_{\mathbf{a}_{2}}^{\perp} \mathbf{a}_{1}$. Clearly, the norm-square of the projection of $\mathbf{a}_{1}$ onto $\mathbf{v}$ is within 0 to $\mathbf{a}_{1}^{H} \mathbf{P}_{\mathbf{a}_{2}}^{\perp} \mathbf{a}_{1} \geq \kappa_{1}$. Using the continuity, there exists a v such that $\mathbf{a}_{1}^{H} \mathbf{v} \mathbf{v}^{H} \mathbf{a}_{1}=\kappa_{1}$.

\section{$4.2 \quad \lambda_{2} \neq 0$}

From (7), we have

$$
\mathbf{w}_{1}=-\frac{\lambda_{1}}{\lambda_{2}} \mathbf{a}_{1} \mathbf{a}_{1}^{H} \mathbf{w}_{1}+\frac{1}{\lambda_{2}} \mathbf{a}_{2} \mathbf{a}_{2}^{H} \mathbf{w}_{1} .
$$

Introducing new variables $c_{1}=-\frac{\lambda_{1}}{\lambda_{2}}$ and $c_{2}=\frac{1}{\lambda_{2}}$ yields

$$
\mathbf{w}_{1}=c_{1} \mathbf{a}_{1} \mathbf{a}_{1}^{H} \mathbf{w}_{1}+c_{2} \mathbf{a}_{2} \mathbf{a}_{2}^{H} \mathbf{w}_{1} .
$$

Under the constraints that $\mathbf{w}_{1}^{H} \mathbf{w}_{1}=1$ and $\mathbf{w}_{1}^{H} \mathbf{a}_{1} \mathbf{a}_{1}^{H} \mathbf{w}_{1}=\kappa_{11}$, we may multiply both sides of (8) by $\mathbf{w}_{1}^{H}$ and $\mathbf{w}_{1}^{H} \mathbf{a}_{2} \mathbf{a}_{2}^{H}$, respectively, and obtain

$$
\begin{aligned}
1 & =c_{1} \kappa_{11}+c_{2} \kappa_{21}, \\
\kappa_{21} & =c_{1} c_{3}+c_{2} \kappa_{21},
\end{aligned}
$$

where $\kappa_{21}=\mathbf{w}_{1}^{H} \mathbf{a}_{2} \mathbf{a}_{2}^{H} \mathbf{w}_{1}, c_{3}=\mathbf{w}_{1}^{H} \mathbf{a}_{2} \mathbf{a}_{2}^{H} \mathbf{a}_{1} \mathbf{a}_{1}^{H} \mathbf{w}_{1}$.

Substituting (8) into $\mathbf{w}_{1}^{H} \mathbf{w}_{1}=1$ and $\mathbf{w}_{1}^{H} \mathbf{a}_{1} \mathbf{a}_{1}^{H} \mathbf{w}_{1}=$ $\kappa_{11}$ gives

$$
\begin{aligned}
c_{1}^{2} \kappa_{11}+c_{2}^{2} \kappa_{21}+2 c_{1} c_{2} c_{3} & =1, \\
c_{1}^{2} \kappa_{11}+\zeta c_{2}^{2} \kappa_{21}+2 c_{1} c_{2} c_{3} & =\kappa_{11}
\end{aligned}
$$

where

$$
\zeta=\mathbf{a}_{1}^{H} \mathbf{a}_{2} \mathbf{a}_{2}^{H} \mathbf{a}_{1}=\cos ^{2} \angle\left(\mathbf{a}_{1}, \mathbf{a}_{2}\right) .
$$

The solutions to $c_{1}, c_{2}, c_{3}$ and the relation between $\kappa_{11}$ and $\kappa_{21}$ are provided in Appendix A. If we follow the same procedure for $\mathbf{w}_{2}$, we will see that the same function is true for $\mathbf{w}_{2}$. Therefore, the channel capacity $R\left(\kappa_{12}, \kappa_{21}\right)$ can be rewritten in terms of $\kappa_{12}$ and $\kappa_{21}$ as

$$
\begin{aligned}
& \frac{1}{2} \log \left(1+\frac{\gamma_{11}\left(\sqrt{\zeta \kappa_{21}}+\sqrt{(1-\zeta)\left(1-\kappa_{21}\right)}\right)^{2}}{1+\gamma_{12} \kappa_{12}}\right)+ \\
& \frac{1}{2} \log \left(1+\frac{\gamma_{22}\left(\sqrt{\zeta \kappa_{12}}+\sqrt{(1-\zeta)\left(1-\kappa_{12}\right)}\right)^{2}}{1+\gamma_{21} \kappa_{21}}\right) .
\end{aligned}
$$

where

$$
\begin{aligned}
\gamma_{i j}=\alpha_{i}^{2} \beta_{j}^{2} & \text { for } i, j=1,2 . \\
0 & \leq \kappa_{12} \leq \zeta \leq 1 \\
0 & \leq \kappa_{21} \leq \zeta \leq 1
\end{aligned}
$$

The channel capacity or the cost function using the substutitions $\kappa_{21}=\cos ^{2}\left(\theta_{2}\right), \kappa_{12}=\cos ^{2}\left(\theta_{1}\right)$, and $\zeta=\cos ^{2}(\phi)$ and performing logarithmic manipulations can be rewritten as

$$
\begin{aligned}
\frac{1}{2} \log \left(\mathbf{J}\left(\theta_{1}, \theta_{2}\right)\right)=\frac{1}{2} \log \{ & \left(1+\frac{\gamma_{11} \cos \left(\theta_{2}-\phi\right)^{2}}{1+\gamma_{12} \cos \left(\theta_{1}\right)^{2}}\right) \times \\
& \left.\left(1+\frac{\gamma_{22} \cos \left(\theta_{1}-\phi\right)^{2}}{1+\gamma_{21} \cos \left(\theta_{2}\right)^{2}}\right)\right\} .
\end{aligned}
$$

The maximum total capacity of a two-user broadcasting system depends on two variables, $\theta_{1}$ and $\theta_{2}$. These variables denote the angles between weight vectors and spatial signatures as seen in (3). From (16), the optimum closed-form solution to this cost function may not exist. This cost function can be evaluated using numerical methods to find global maximum. It is seen from (16), for certain $\kappa_{11}$ and $\kappa_{22}, R$ is maximum. Therefore, the search for optimal weight vectors can be accomplished by the following steps

1. For a given $\left(\kappa_{12}, \kappa_{21}\right)$ pair, find the legitimate $\mathbf{w}_{1}$ which maximizes $\cos ^{2} \angle\left(\mathbf{w}_{1}, \mathbf{a}_{1}\right)$ and $\mathbf{w}_{2}$ which maximizes $\cos ^{2} \angle\left(\mathbf{w}_{2}, \mathbf{a}_{2}\right)$. Evaluate $R\left(\kappa_{12}, \kappa_{21}\right)$. 
2. Identify the optimal pair $\left(\kappa_{12, \text { opt }}, \kappa_{21, \text { opt }}\right)$ which maximize $R\left(\kappa_{12}, \kappa_{21}\right)$.

3 . The weight vectors which corresponding to $\left(\kappa_{12, \text { opt }}, \kappa_{21, \text { opt }}\right)$ in step 1 are the solutions.

Step 2 involves only a two-dimensional searching, which is tractable in general; however, we will derive a near-optimum solution to build up some intuition or understanding on the system behavior by using sensitivity analysis in the next Section.

\section{Near-Optimum Closed-Form Solu- tion}

A good initial guess at the optimum value is $\theta_{1}=\phi$ and $\theta_{2}=\phi$, which are the left endpoints of $\kappa_{12}$ and $\kappa_{21}$, respectively, because they bring the cosine terms in the numerators in (16 to 1 . . To refine this solution, we will perform sensitivity analysis around this operating point by varying $\theta_{1}$ and $\theta_{2}$ slightly using $\theta_{1}=\phi+\triangle \theta_{1}$ and $\theta_{2}=\phi+\triangle \theta_{2}$. Then, the cost function becomes

$$
\begin{aligned}
\mathbf{J}\left(\triangle \theta_{1}, \triangle \theta_{2}\right)= & \left(1+\frac{\gamma_{11} \cos ^{2}\left(\triangle \theta_{2}\right)}{1+\gamma_{12} \cos ^{2}\left(\phi+\triangle \theta_{2}\right)}\right) \times \\
& \left(1+\frac{\gamma_{22} \cos ^{2}\left(\triangle \theta_{1}\right)}{1+\gamma_{21} \cos ^{2}\left(\phi+\triangle \theta_{1}\right)}\right) .
\end{aligned}
$$

\subsection{Simplest Small Angle Assumptions}

Small angle assumptions for $\triangle \theta_{1}$ and $\triangle \theta_{2}$ :

$$
\begin{aligned}
& \sin \left(\phi+\triangle \theta_{i}\right)=\sin (\phi) \\
& \cos \left(\phi+\triangle \theta_{i}\right)=\cos (\phi)
\end{aligned}
$$

and

$$
\begin{aligned}
& \sin \left(\triangle \theta_{i}\right)=\Delta \theta_{i} \\
& \cos \left(\triangle \theta_{i}\right)=1
\end{aligned}
$$

where $i=1,2$. If we solve $\mathbf{J}\left(\triangle \theta_{1}, \triangle \theta_{2}\right)$ for $\left(\triangle \theta_{1}, \triangle \theta_{2}\right)$ using the assumptions in (18) and (19)

$$
\left\{\triangle \theta_{1}:\left.\frac{\partial \mathbf{J}\left(\triangle \theta_{1}, \triangle \theta_{2}\right)}{\partial \triangle \theta_{1}}\right|_{\triangle \theta_{2}=0}=0\right\}
$$

and

$$
\left\{\triangle \theta_{2}:\left.\frac{\partial \mathbf{J}\left(\triangle \theta_{2}, \triangle \theta_{1}\right)}{\partial \triangle \theta_{2}}\right|_{\triangle \theta_{1}=0}=0\right\}
$$

we get

$$
\triangle \theta_{i}=\frac{\gamma_{j i} \gamma_{j j} \cos (\phi) \sin (\phi)\left(1+\gamma_{i i}+\gamma_{i j} \cos ^{2}(\phi)\right)}{\gamma_{i i}\left(1+\gamma_{j i} \cos ^{2}(\phi)\right)\left(1+\gamma_{j j}+\gamma_{j i} \cos ^{2}(\phi)\right)}
$$

where $i, j=1,2$ and $i \neq j$. This assumption performs well for many cases; however, it is sensitive to the value of $\zeta=\cos ^{2}(\phi)$. To overcome this problem we introduce a more complete small angle assumption.

\subsection{Improved Small Angle Assumption}

Two of the small angle assumptions are not correct:

$$
\begin{aligned}
& \cos \left(\phi+\triangle \theta_{i}\right)=\cos (\phi)-\triangle \theta_{i} \sin (\phi) \\
& \sin \left(\phi+\triangle \theta_{i}\right)=\sin (\phi)+\triangle \theta_{i} \cos (\phi)
\end{aligned}
$$

We will still hold ( $\triangle \theta_{1}, \triangle \theta_{2}$ ) to be a small constant value for these two relationships. Using the equations in (20), (21), and with the assumptions (18), (23), and (19), we get the similar result to (22) but scaled by $2 \zeta=2 \cos ^{2}(\phi)$. Thus, the near optimum angle between the $i$ th weight vector and the $j$ th spatial signature vector becomes

$$
\theta_{i}=\phi+2 \cos ^{2}(\phi) \triangle \theta_{i}
$$

Using the closed-form solutions in (24), the nearoptimum weight vectors can be found by the following steps

1. For a given $\left(\mathbf{a}_{1}, \mathbf{a}_{2}\right)$ spatial signature pair, find the $\zeta=\cos ^{2}(\phi)$ from (13).

2. Compute $\gamma_{i j}$ for $\left(\alpha_{i}, \beta_{j}\right)$ from (15).

3 . Find the near-optimum angles using (24) and (22).

4. The weight vectors which corresponding to $\left(\theta_{1}, \theta_{2}\right)$ are the solutions.

\section{$6 \quad$ Numerical Examples}

In this section, a numerical example is presented to compare numerical search method and near-optimum closed form solution. We also plot the single antenna capacity and naive time sharing performance. In the numerical example, we consider the case where we have a linear uniform array with 8 antenna elemets transmitting to two users. In the example, the angle between spatial signatures and the gains of spatial signatures are $\phi=54, \alpha_{1}=1.22$, and $\alpha_{2}=1.333$.

\section{Conclusion and Future Directions}

In this paper, the channel capacity of a two-user, multi-transmitter broadcast system were discussed. A near-optimum closed form solution for designing the weight vectors was presented. This solution was derived by means of sensitivity analysis. Designing of the optimal weight vectors $P$-user case $(P>2)$ is currently under investigation. 


\section{Appendix A}

Through straightforward derivation, we can solve the four real variables $c_{1}, c_{2}, c_{3}$ and $\kappa_{11}$ from (9) to (12). In particular, $c_{2}$ are the roots of the following second-order equation,

$$
\left(\frac{\kappa_{11}}{1-\zeta}-1\right) c_{2}^{2}+\frac{2\left(1-\kappa_{11}\right)}{1-\zeta} c_{2}-\frac{\left(1-\kappa_{11}\right)}{(1-\zeta)^{2}}=0
$$

and

$$
\kappa_{21}=\frac{1-\kappa_{11}}{(1-\zeta) c_{2}^{2}} .
$$

Equation (A.1) has roots which are

$$
\begin{aligned}
& c_{2,1}=\frac{(1-\zeta)\left(1-\kappa_{11}\right)+\sqrt{\zeta \kappa_{11}(1-\zeta)\left(1-\kappa_{11}\right)}}{(1-\zeta)\left(1-\zeta-\kappa_{11}\right)} \\
& c_{2,2}=\frac{(1-\zeta)\left(1-\kappa_{11}\right)-\sqrt{\zeta \kappa_{11}(1-\zeta)\left(1-\kappa_{11}\right)}}{(1-\zeta)\left(1-\zeta-\kappa_{11}\right)}(
\end{aligned}
$$

where $c_{2,1}$ in (A.2) should be used to estimate $\kappa_{21}$ because the second expression provides the length that is shown in Figure 1 . Thus, $\kappa_{21}$ can be expressed as

$$
\kappa_{21}=\frac{\left(1-\zeta-\kappa_{11}\right)^{2}}{(1-\zeta)\left(1-\kappa_{11}\right)+2 \sqrt{\zeta \kappa_{11}(1-\zeta)\left(1-\kappa_{11}\right)}+\zeta \kappa_{11}}
$$

Using this relation, we can find the maximum value of $\kappa_{11}$ when $\kappa_{21}$ is given. That is,

$$
\kappa_{11}=\left(\sqrt{\zeta \kappa_{21}}+\sqrt{(1-\zeta)\left(1-\kappa_{21}\right)}\right)^{2} \text {. }
$$

\section{References}

[1] P. Balaban and J. Salz, "Optimum Diversity Combining and Equalization in Digital Data Transmission with Applications to Cellular Mobile Radio - Part I: Theoretical Considerations," IEEE Trans. on Communications, 40(5):885-894, May 1992.

[2] P. Balaban and J. Salz, Optimum diversity combining and equalization in data transmission with application to cellular mobile radio - part ii: Numerical results, IEEE Trans. Communication, Com-40(5):895907, May 1992.

[3] S. U. Pillai, Array Signal Processing, Springer Verlag, New York, 1989.

[4] T.M. Cover and J. Thomas, Elements of Information Theory, John Wiley \& Sons, Inc., New York, NY, 1991.

[5] G. Xu, H. Liu, W. Vogel, H. Lin, S.S. Jeng, and G. Torrence, "Experimental Studies of Space-DivisionMultiple-Access Schemes for Spectral Efficient Wireless Communications," Proc. ICC'94, pp. 800-804, May 1994.
[6] B. Suard and G. Xu and H. Liu and T. Kailath, "Channel capacity of space-division-multiple-access schemes", Proc. 28 $8^{t} h$ Asilomar Conference on Signals, Systems and Computers, Pacific Grove, CA, pp. 1159-1163, Nov. 1994

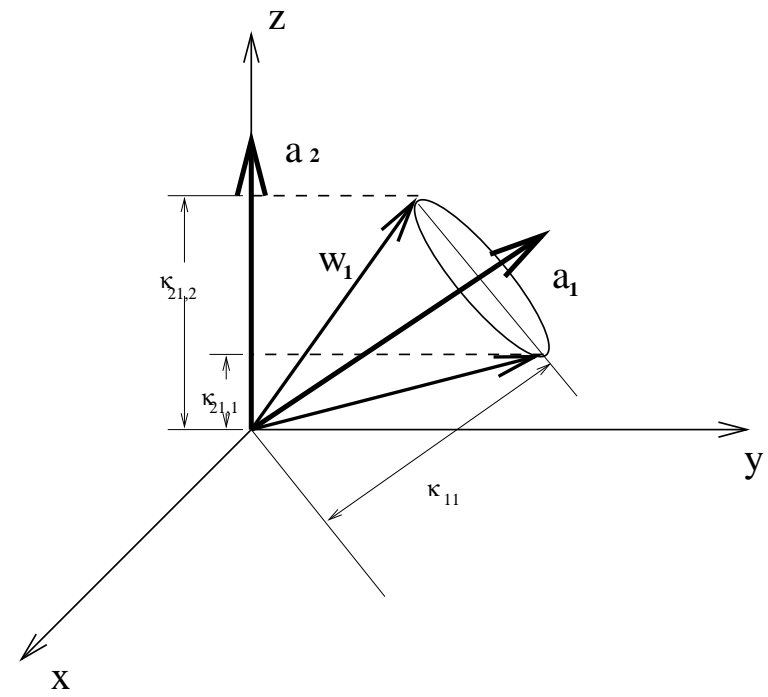

Figure 1: Illustration of search for a optimal weight vector

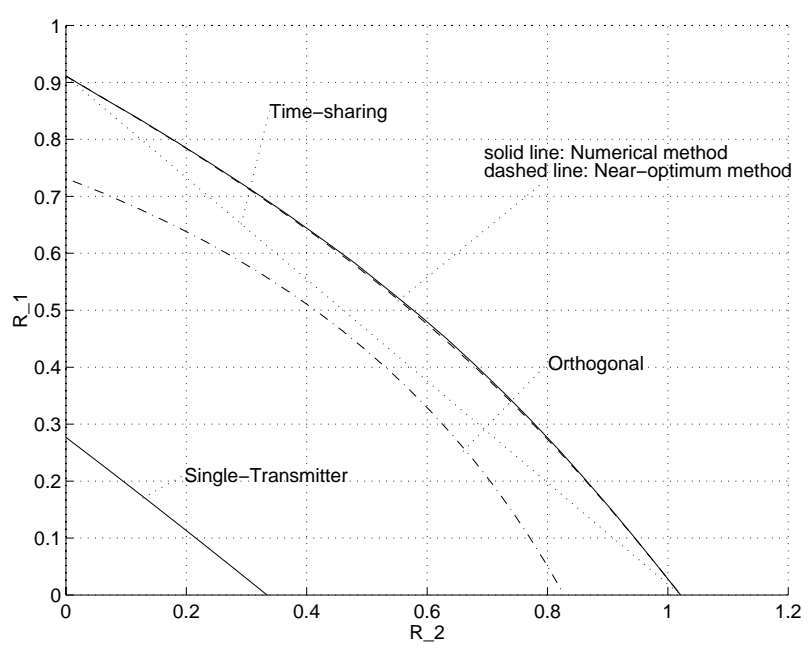

Figure 2: Channel capacities using different weight vectors 